studies will serve in research of utilization of this toxic substance.

\section{SUMMARY}

Biological activities of a new toxic substance, Teleocidin, produced by a Streptomyces were examined. Among the toxic antibiotics produced by Streplomyces, 9 antibiotics were also tested for their toxicity toward Oryzias latipes, Daphnia magna and Bugula neritina larvae, and Antimycin A was found to show equal or more severe toxicity than Teleocidin.

Teleocidin has shown certain inhibitory actions to Ascaris suilla and Turbatrix aceti and also has severe toxicity and skin irritative effects on mammalians.
Acknowledgements. The authors wish to express their sincere gratitude to Dr. K. Sakaguchi and Prof. K. Arima of Tokyo University, and to Mr. T. Fujisawa, Dr. K. Ueda and Dr. T. Fujisawa of this company for their kind guidance throughout this work. Thanks are also due to Prof. T. Bando of Juntendo University, Assist. Prof. T. Hibiya, Assist. Prof. T. Yamazaki, Mr. R. Hirano of Tokyo University and members of this laboratory for their kind instruction and suggestions concerning the studies on toxicity.

They also wish to express their indebtness to Assist. Prof. H. Yonehara, Institute of Applied Microbiology, University of Tokyo, for kindly supplying the antibiotics used in this work.

[Bull. Agr. Chem. Soc. Japan, Vol. 24, No. 4, p. 655 657, 1960]

\title{
Studies on the Reduction of Terpenes with Sodium in Aqueous Ammonia
}

\author{
Part XI. On the Reduction of Isopulegone* \\ By Hiroo UEDA \\ Research Institute, Nagaoka and Co., Ltd., Motoyama, Kobe \\ Received May 13, 1960
}

\begin{abstract}
On the reduction of isopulegone with sodium in aqueous ammonia, it was found that the main product was isopulegol. This fact indicates that the double bond not in conjugation with carbonyl group is not attacked.
\end{abstract}

In the previous papers, it has been shown that saturated ketones $\left((-)\right.$-menthone ${ }^{1)},(-)$-isomenthone $^{2\rangle},( \pm)$-menthone ${ }^{3)},( \pm)$-isomenthone ${ }^{3)}$,

* For Part X see This Bulletin, 24, 601 1960i.

1) H. Ueda, Y. Shibahara and S. Shimizu, This Balletin, 23, 376 (1959).

2) H. Ueda, ibid., 24, $595(1960)$.

3) H. Ueda, ioid., 24, 505 (1950). inverted menthone $\left.{ }^{4)}\right), \alpha, \beta$-unsaturated ketones $\left((-)\right.$-carvone ${ }^{5)},(+)$-pulegone ${ }^{6)},( \pm)$-piperitone $\left.{ }^{7)}\right)$ and cross conjugated ketone (piperitenone ${ }^{3)}$ )

4) H. Ueda and S. Shimizu, ibid., 23, 380 (1959).

5) H. Uedi and S. Shimizu, ibid., 23, 524 (1959).

6) H. Ueda and S. Shimizu, ibid., 24, 92 (1960).

7) H. Ueda, ibid., 24, $598(1960)$.

8) H. Ueda, ithd., 24, 601 (1960.. 
yielded saturated alcohols by the treatment with sodium in aqueous ammonia, i.e., a novel method of reduction employed in this series.

The present investigation was undertaken to determine the composition of the reduction product of isopulegone ( $\beta, \gamma$-unsaturated ketone), on the same reduction as described above.

In the reduction of carvone, the carbonyl group and double bond conjugated with the carbonyl group in carvone were reduced, but the unconjugated double bond was not affected, dihydrocarveol being produced ${ }^{5}$. In view of this result, it is presumed that isopulegone would be reduced with sodium in aqueous ammonia to give isopulegol.

On the other hand, from the fact that isopulegone is easily converted to pulegone by the treatment with sodium-ethoxide or baryta ${ }^{97}$, it is also possible to suppose that isopulegone would be converted by alkali in reaction medium to pulegone, which would be reduced to menthol, as reported previously ${ }^{6)}$.

The experiment described below was undertaken in order to make a choice between these alternative presumptions.

The experiment showed that the reduction product of isopulegone consisted of $90 \%$ of unsaturated alcohol and 10\% of menthol. The unsaturated alcohol was characterised mainly as (-)-isopulegol by the bromide of its 3,5dinitrobenzoate. Catalytic hydrogenation of the reduction product gave (-)-menthol predominantly.

This showed that the majority $(90 \%)$ of isopulegone is reduced to isopulegol by the treatment with sodium in aqueous ammonia, though a minor amount (10\%) give menthol through pulegone.

\section{EXPERIMENTAL}

All melting and boiling points were uncorrected. Ultra violet spectra were determined at Prof. Shimizu's Laboratory, Shinshu University.

\section{Isopulegone :}

9) J. L. Simonsen, "The Terpenes" Cambridge Univ. Press. 1953. Vol. I. p. 389.
Isopulegol, prepared from citronella ${ }^{10)}$, b.p. $69.0^{\circ} / 6.5$ $\mathrm{mm}, d_{4}^{18.5} 0.9148, n_{\mathrm{D}}^{18.5} 1.4731$, MR 47.31 (Calcd. 47.24), $[\alpha]_{D}^{18.5}-1.0^{\circ}$ (homog.), was oxidised by sodium dichromate in acetic acid at $60^{\circ}$. The oxidation product was a colorless oil, which was confirmed to be composed mainly of isopulegone containing a trace amount of pulegone by assay of its ultraviolet spectrum (pure pulegone had $\lambda_{\text {max }}^{\mathrm{MenH}} 253 \mathrm{~m} \mu, E_{\max }^{\mathrm{MeOH}} 7500^{11)}$ ); Yield $74.1 \%$, b.p. $72.5^{\circ} / 7 \mathrm{~mm}, d_{4}^{20} 0.9158, n_{\mathrm{D}}^{20} 1.4696$, MR 46.34 (Calcd. 45.72), $[\alpha]_{\mathrm{D}}^{20}-3.7^{\circ}$ (homog.), $\lambda_{\max }^{\mathrm{MeOt}} 253$ $\mathrm{m} \mu, \mathrm{E}_{\mathrm{mix}}^{\mathrm{Me}} \mathrm{NH} 137$.

\section{Reduction of isopulegone:}

Isopulegone $(10.0 \mathrm{~g})$ was reduced with sodium $(7.5 \mathrm{~g})$ in aqueous ammonia and $n$-hexane (reaction temp. $0 \sim 3^{\circ}$ ), in the same way as previously reported". The reduction product $(9.8 \mathrm{~g}$, Yield $96.7 \%)$ was a colorless oil, and did not crystallise on cooling $\left(-22^{\circ}\right)$; b.p. $73 \sim$ $75^{\circ} / 7 \mathrm{~mm}, d_{4}^{20} 0.9028, n_{\mathrm{D}}^{20} 1.4698$, MR 47.71 (Calcd. $47.24),[\alpha]_{\mathrm{D}}^{20}-12.0^{\circ}$ (homog.), $\lambda_{\mathrm{max}}^{\mathrm{MeNI}} 268 \mathrm{~m} \mu, \mathrm{E}_{\mathrm{max}}^{\mathrm{MeOH}} 4$.

Identification of (-)-isopulegol :

The reduction product $(2.07 \mathrm{~g})$ gave crude 3,5 -dinitrobenzoate, m.p. $85 \sim 101^{\circ}$, in a quantitative yield. Two crystallisations of its crude ester from $n$-hexane gave pale yellow needies $(2.62 \mathrm{~g}$, Yield $56.1 \%$ based on the reduction product), m.p. 103 109 ${ }^{\circ}$, iodine-number 62.80 . The iodine-number was determined by Rosemund. Kuhnhenn's method, using pyridine-bromine-sulphate ${ }^{\text {s) }}$, and it indicated that its 3,5-dinitrobenzoate fraction contained $86.2 \%$ of unsaturated ester. One crystallisation. from $n$-hexane of the crude bromide $(310 \mathrm{mg})$, recovered from titrated solution of iodine-number determination of its 3,5-dinitrobenzoate $(201 \mathrm{mg})$, gave (-)-dibromoisopulegyl 3,5-dinitrobenzoate (178 mg, Yield $70.5 \%$ for theor.), m.p. $110^{\circ}$, undepressed by admixture with an authentic sanple. The authentic (-)-dibromo-isopulegyl 3,5-dinitrobenzoate, m.p. $110^{\circ}$, was prepared from $(-)$ isopulegyl 3,5-dinitrobenzoate, m.p. 104 104.5 10), in. the same way as described above.

Catalytic $c_{2}^{\top}$ hydrogenation of the reduction product:

The reduction product $(3.48 \mathrm{~g})$ was hydrogenated over a platinum oxide catalyst $(50 \mathrm{mg})$ in acetic acid. It absorbed $489 \mathrm{ml}\left(21^{\circ} \mathrm{C}\right)$ of hydrogen, equivalent to $89.8 \%$ of the theory on the basis of isopulegol, and gave menthol mixture $3.13 \mathrm{~g}$, Yield $88.7 \%$ for theor.); b.p. $80 \sim 81^{\circ} / 6$ mm, m.p. $22 \sim 23.5^{\circ}, d_{4}^{25} 0.8893, n_{\mathrm{D}}^{25} 1.4580$, MR 47.94 (Calcd. 47.71), [a. $]_{\mathrm{D}}^{25}-29.9^{\circ}$ (homog.). The menthol mixture (496 mg) gave (-)-menthyl 3,5-dinitrobenzoate

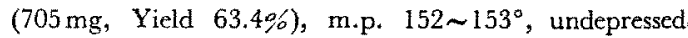

10) H. Ueda and S. Shimiza, This Bulletin, 24, 402 (1960).

11) S. Shimizu, J. Sbinsbu Unit. Japan* No.4, 73 (1954)* 
by admixture with an authentic sample ${ }^{123}$.

Acknowledgements The author is indebted to Emeritus Prof. S. Takei, Prof. T. Mitsui and Prof.

12) H. Ueda and T. Mitsui, J. Agr. Chem. Sor. Japan, 28, 945 (1954).
M. Nakajima, University of Kyoto, for their kind advices and suggestions. He also wishes to express his sincere thanks to Prof. S. Shimizu, Shinshu University, and Dr. Y. Inouye, Institute for Chemical Research, University of Kyoto, for their kind guidances throughout this series.

[Bull. Agr. Chem. Soc. Japan, Vol. 24, No. 7, p. 657 666, 1960]

\title{
Molecular Complexes of Tetracycline Acid Salts
}

\section{Part I. The Preparations and Properties of Tetracyclinium Salt-Oxalic Acid Complexes*}

\author{
By Hiroshi Ogawa and Shigeharu Inouye \\ Research Laboratory, Meiji Seika Co. Ltd., Horikawa-cho, Kawasaki \\ Received May 19, 1960
}

\begin{abstract}
A series of studies on the crystalline molecular complexes of tetracycline groups have been carried out in this laboratory, and the present paper deals with the compositions of the oxalic acid complexes of various tetracycline acid salts, which were examined by X-ray powder diffraction methods, infrared spectra, and chemical analyses. Insertions of salt anions and crystal water in the crystal lattice of the complexes, and the important role of the latter for crystallization are indicated.
\end{abstract}

Broad-spectrum antibiotic tetracycline (TC), which was first obtained from catalytic reduction of 7-chlorotetracycline ${ }^{1)}$ and later by the direct fermentation of a Streptomyces ${ }^{2)}$, shows interesting physico-chemical behaviors different from other acid and basic antibiotics.

Some properties of $\mathrm{TC}$ are attributable to its amphoteric character including unusual insolu-

* S. Inouye and H. Ogawa, The paper presented at tbe Kanto Local Meeting of the Agricultural Chemical Society of Japan, Feburuary 27, 1960.

1) J. H. Boothe, J. Morton, J. P. Petisi, R. G. Wikkins and J. H. Williams, J.Am. Cbem. Soc., 75, 4621 (1953); W. T. Moreland, A. R, English, C. R. Stephens and F.J. Pilgrim, J. Am. Cbem. Soc., 75, 4622 (1953).

2) H. Ogawa and T. Ito, J. Agr. Chem. Soc. Japan, 30, 123 (1956); P. P. Minieri, H. Sokol and M. C. Firman, U. S. Patent No. 2,734,018 (1956). bility in neutral aqueous solution. Due to these properties, TC hydrochloride is generally used clinically and its alkaline earth metal salts are usually applied for the precipitation of TC from the fermentation broths owing to the sparing solubility of these salts. Aromatic sulfonic acid salts of TC have also been reported ${ }^{3}$.

Furthermore, the strong polarity and the polyfunctional character of $\mathrm{TC}$ may suggest the formation of complexes having different solubilities. Indeed, many complexes of tetracyclines have been described in the literatures as follows: Calcium chloride complex of 5-

3) F.A. Marcketti and G. Casini, Ricerca. scie., 26, 3321 (1956). 